\title{
Human Creativity and Artificial Intelligence (AI): Two Distinct Requirements for Sustainable Competitive Advantage
}

\author{
Nima S. Salami, faculty \\ NimaSalami@westcliff.edu
}

\begin{abstract}
Increasing competition among industries requires them to be intelligent in order to make their best decisions and remain competitive in the market. Nowadays both human and artificial intelligence have made many contributions to this purpose and have helped businesses tremendously to achieve their goals. This paper explains why industries need to realize that intelligence and creativity are two distinct disciplines, and each needs to be treated differently in combination with artificial intelligence (Al) in order to maintain a sustainable competitive advantage in the future.
\end{abstract}




\section{INTRODUCTION}

Companies that are able to formulate effective strategies would be able to outperform their competition and remain at a superior position in the market when compared to others (Rothaermel, 2015). If a company is able to continuously maintain its superiority for a prolonged period of time, it will have gained a sustainable competitive advantage over other rivals (Rothaermel, 2015). The mainstream idea is that the formulation of such strategy requires management to have relevant talent and intelligence in order to execute the devised strategy both properly and successfully (Thompson, Strickland, Gamble, \& Zeng'an Gao, 2008). Interestingly it was not until mid-50s that researchers finally started to distinguish between intelligence and creativity as separate phenomena (Proctor, 2014).

On the other hand, artificial intelligence (AI) has been around since 1955, the year that it was introduced to the market (Socha, 2017). Al is defined as the ability of digital machines to make calculations and decisions and learn, faster than what humans are capable of (Socha, 2017). Since then, Al has been used in unstructured strategic decision-making processes, in simulating human thinking, in reasoning and learning, in learning or understanding from experiences, in making sense of contradictory information, and in making effective and efficient decisions for organizations (Baltzan \& Phillips, 2015).

As a result, when Deep Blue, a chess-playing computer developed by IBM, defeated Gary Kasparov in the world class champion chess competitions, the strength with which the machine was able to compete in the competition was interpreted as intelligence (Newborn, 2012). Therefore, at that time period, it was safe for people to assume that the winners of these types of logical games, are those who have higher levels of intelligence, with either higher IQs or stronger analytical skills (Proctor, 2014).

In this paper, moderating factors to curate a sustainable competitive advantage such as human creativity, innovation, creativity, intelligence, and artificial intelligence will be discussed and analyzed.

\section{Human CREATIVITY}

Creativity has been a popular topic recently especially in the high-tech industries (Proctor, 2014; Schilling, 2017). Although there have been several definitions for the term, there still exists much confusion about what creativity means exactly (Proctor, 2014). For instance, Maslow (1954) considered that creativity has two levels, primary and secondary. In the former, an individual's new discoveries, novel thinking, and new insights are important. Whereas, in the latter, the collective characteristics of collaborative discoveries and works of scientists extends from previous works and creates new insights.

Although there is a lack of consensus between researchers in defining creativity (Parkhurs, 1999), many of them agree that creativity is directly related to the newness and usefulness of an idea (Mumford, 2003). Batey (2012) adds that any product resulting from creativity should be novel, useful, and original in order to be considered in the framework.

\section{Innovation and invention}

Schilling (2017) believes that innovation is the practical product of creativity that has been used in devices or processes. Proctor (2014) has furthered this concept to advocate that innovation needs to create a significant change in the application of the technology as well. He has further explained that there is a distinction between invention and innovation, where the former is "the formulation of a new idea" but the latter "concerns the practical application of new inventions into marketable products or services" (Proctor, 2014, p. 4).

Moreover, it has been argued that since many of the situations that have arisen in today's business environments are unique, have no precedence, or history, organizations that have inherited creativity and continuous innovation in their cultures are the ones that can overcome these situations and sustain their competitive advantage (Proctor, 2014). Therefore, creative thinking, innovation, and creative problem-solving abilities 
for all members of an organization, specifically for the management team, would be essential (Proctor, 2014).

On the other hand, research has shown that an organization's internal and external environments, including economic, political, technological, social, and cultural forces can produce opportunities as well as threats for the company. If managers do not recognize them nor continuously look for creative solutions, it might be too late to react, and the company might lose its competitive advantage in the market and among the competition (Large, 1992). In a famous case where, negative social forces were involved, Air Canada's logo was negatively associated by customers with the government's defective bureaucracy. For this reason, the sales went down (Proctor, 2014). Managers started to look for the sources of the problem, and eventually, their surveys determined why customers were reluctant to purchase tickets from them. The creative solution was to implement a subtle change to the company's logo design. This small change, using the creative approach crafted by designers, was successful and was accepted by the customers. The quick remedy for the business's problem resulted in the continuation of the company, the employees' jobs, and their overall reputation (Proctor, 2014).

\section{Individual creativity}

Research has shown that an individual's creativity is a collective function of factors such as personality, intellectual abilities, knowledge, style of thinking, environment, and motivation (Sternberg, \& Lubart, 1999). Schilling (2017) suggests that certain intellectual abilities can increase creativity. These abilities include how individuals perceive issues in a different capacity, how they pick related problems that they deem to be worth pursuing, and how they explain and articulate the new ideas to others in an attempt to convince them (Schilling, 2017).

Interestingly, Schilling (2017) has argued that the effect of knowledge on creativity is doubleedged, where in extreme situations, individuals' creativity will decrease in cases where the knowledge they possess is either lacking or is too high. For instance, if an individual has a lot of knowledge in a specific field, he/she could be trapped in his/her paradigms, existing logic, and perceptions, therefore decreasing creativity (Schilling, 2014). Also, if he/she does not have enough knowledge about the subject matter, his/her understanding would not be enough to produce a meaningful result. Therefore, again his/her creative solutions could potentially be worthless (Proctor, 2014). Therefore, a moderate knowledge of a field is optimum to produce the maximum number of creative solutions for individuals (Schilling, 2017).

Proctor (2014) has also suggested that an individual's creativity could be blocked if certain unfavorable conditions such as mindsets, emotions, perceptions, expressions, and cultural influences are present and can block individuals from creativity. By definition, a mindset is an individual's over-sensitized bias for particular parts of the information, where his/her feelings might exclude some portions of necessary information, which can create a block (Proctor, 2014).

\section{Organizational creativity}

Woodman, Sawyer, and Griffin (1993) have suggested that organizational creativity is the function of all individuals' creativities, social processes, and contextual factors within an organization. They argue that organizational creativity is not the aggregate creativity of all members combined. This notion makes it clear that organizations not only need to have creative people on board, but they also should create the proper environment, processes, and contextual factors that would foster and encourage creativity (Schiller, 2017).

For instance, methods such as suggestion boxes, idea management systems (database for uploading ideas and comments for everyone's review), employee driven idea system (an online platform for suggestions, but if accepted, are implemented by the same employee), and creativity training programs (in which managers learn how to verbally and nonverbally appreciate employees' creative thinking) can be used to increase organizational creativity (Shilling, 2017).

Other techniques such as, "one great idea" (enables individuals to have access to a company's 
idea depository through the intranet), developing alternative scenarios, comparative analogies for problem solving, creating mock prototypes of potential new products, and playful design of product features, have all proved to be beneficial for organizational creativity (Schilling, 2017).

\section{Intelligence Versus CReativity}

More intelligent people can naturally score higher on intelligence tests since they have larger working memories compared to other people which enable them to quickly find answers to complex problems (Proctor, 2014). However later studies have not been able to find a correlation between intelligence and creativity, and therefore, psychologists believe that intelligence and creativity are two distinct phenomena (Proctor, 2014). For instance, there can be people in an organization that are highly intelligent, but are not highly creative and vice versa (Proctor, 2014).

Intelligent people are more likely to use reproductive thinking, which is the use of previous experiences, to make comparisons with similar problems that they have encountered in the past in order to find quick conventional solutions for their problems (Proctor, 2014). Research has shown that this type of thinking generates rigidity in thought and only serves to consolidate the paradigms and mindsets of individuals, thus, leading to creativity blocks (Proctor, 2014).

On the other hand, creative people are more likely to think productively. This term refers to the ability to look into a problem from many different perspectives and identify multiple potential solutions (Proctor, 2014). These creative productive thinkers have the ability to form many novel combinations of solutions for the identified problems and identify the juxtaposition between dissimilar subjects to resolve issues (Proctor, 2014).

In productive thinking processes, the creative mind will consider all possible solutions for the problem including both the obvious and obscure ones, even after a promising solution has been found for the problem (Proctor, 2014).

Productive thinking styles are crucial forms of creativity for all businesses since these techniques can generate more and more solutions for the same problems. This can increase the likelihood of finding a creative solution for the issue and most probably will help the company to sustain its competitive advantage (Proctor, 2014). It has been explained that highly productive thinking individuals have the ability to tolerate the ambivalence that might exist between the opposites, or two entirely incompatible subjects (Proctor, 2014). For instance, the same abilities for tolerating the ambivalence made it possible for Thomas Edison to invent electric lamp bulbs, as he could tolerate to combine two ambivalent objects (high-resistance filaments and high resistant parallel wiring circuits). This was something that could easily look impossible to many conventional reproductive thinkers (Proctor, 2014).

\section{Artificial intelligence (AI)}

Artificial intelligence (Al) is a digitized computational technology that enables managers to make more effective and efficient decisions (Baltzan \& Phillips, 2015). There are five categories of Al systems; expert systems, neural networks, generic algorithms, intelligent agents, and virtual reality (Baltzan \& Phillips, 2015). These systems can increase the speed, consistency, and problemsolving abilities (where available information is incomplete). Additionally, they are able to resolve complicated issues and boost the productivity of assembly lines, resulting in more effectiveness and efficiency (Baltzan \& Phillips, 2015).

The above capabilities can easily enhance a company's competitive advantage as they make an organization more effective and efficient. However, there is no guarantee for the sustainment of competitive advantage. These systems can help automation, streamlining (removing bottlenecks and redundancies), and business reengineering processes (to find a totally different approach) (Baltzan \& Phillips, 2015). Business process reengineering (BPR) systems, together with management information systems (MIS), are the only Al systems that can enhance managers' creative problem-solving capabilities to sustain the competitive advantage (Baltzan \& Phillips, 2015).

If managers are not creative thinkers or creative problem solvers, the combination of the above Al capabilities (specifically BPR and MIS) 
would not render the best results for an organization (Baltzan \& Phillips, 2015). Therefore, it becomes crucial for managers to be able to think outside of the box and possess the ability to focus on the core strategic processes of their organizations that are critical for performance, rather than focus on managerial functions that have little impact on their organizational processes (Baltzan \& Phillips, 2015).

For instance, Progressive, an insurance company, was able to innovatively make fundamental changes in its processes with the help of its BPR and MIS (Baltzan \& Phillips, 2015). The company was able to reduce the settlement time for its standard claims from 31 days to four hours (Baltzan \& Phillips, 2015). Progressive's use of BPR in innovation entirely reshaped and redefined the methods of the whole industry (Baltzan, \& Phillips, 2015).

\section{CONCLUSIONS}

In today's dynamic nature of the markets, sustaining a competitive advantage is a must (Rothaermel, 2015). Sustainable competitive advantage is attainable through the development of a thorough comprehensive strategy that has been formulated by expert, talented, and creative managers, executed by employees and reviewed consistently (Thompson et al., 2008). Although intelligent managers and employees are required for the success of any organization, if they are not creative problem-solvers, do not have innovative personalities, or are not novel thinkers, they may not be able to develop the most efficient strategies for their organizations. Additionally, they would not be able to resolve complex unprecedented issues and even the companies' best opportunities may be overlooked or even lost (Proctor, 2014).

If managers are not creative thinkers, even artificial intelligence and information systems such as BPR and MIS would not be effective nor efficient for their organizations. This lack of efficiency would waste the company's resources (Baltzan \& Phillips, 2015). Therefore, any organization's solution for developing a sustainable competitive advantage would result from a set of strong, intelligent, creative, and innovative employees whose abilities are reinforced by their organization's supportive and rewarding culture and environment. Such an organization would be able to use its Al capabilities and sustain its competitive advantage both effectively and efficiently. 


\section{REFERENCES}

Baltzan, P., \& Phillips, A. (2015). Business driven information systems. McGraw-Hill/Irwin.

Batey, M. (2012). The measurement of creativity: From definitional consensus to the introduction of a new heuristic framework. Creativity Research Journal, 24(1), 55-65.

Large, M. (1992). Eco-mapping-how to avoid boiled frogs. Management Education and Development, 23(4), 317325.

Maslow, A. H. (1954). Personality and motivation. Harlow, England: Longman, 1, 987.

Mumford, M. D. (2003). Taking stock in taking stock. Creativity Research Journal, 15(2-3), 147-151.

Newborn, M. (2012). Kasparov versus Deep Blue: Computer chess comes of age. Springer Science \& Business Media.

Parkurst, H., B. (1999). Confusion, lack of consensus, and definition of creativity as a construct. The Journal of Creative Behavior, 33, 1-21.

Proctor, T. (2014). Creative Problem Solving for Managers: Developing Skills for Decision Making and Innovation. Routledge.

Rothaermel, F. T. (2015). Strategic management. New York: McGraw-Hill Education.

Schilling, A. S. (2017). Strategic Management of Technological Innovation. New York: McGraw-Hill.

Socha, G. (2017). What will Al mean for you? Judicature, 101(3), 6-8. Retrieved from https://search.proquest.com/docview/2102830485?accountid=158986

Sternberg, R. J., \& Lubart, T. I. (1999). The concept of creativity: Prospects and paradigms. Handbook of creativity, 1, 3-15.

Thompson, A. A., Strickland, A. J., Gamble, J. E., \& Zeng'an Gao. (2008). Crafting and executing strategy: The quest for competitive advantage: Concepts and cases (19 $19^{\text {th }}$ ed.).

Woodman, R. W., Sawyer, J. E., \& Griffin, R. W. (1993). Toward a theory of organizational creativity. Academy of Management Review, 18(2), 293-321. 